\title{
MIMO precoding and mode adaptation in femtocellular systems
}

\author{
Chenzi Jiang ${ }^{1 *}$, Leonard J Cimini $\mathrm{Jr}^{1}$ and Nageen Himayat ${ }^{2}$
}

\begin{abstract}
Hierarchical femtocellular architectures have become popular recently because of their potential to provide increased coverage and capacity in cellular systems. However, introduction of femtocells might reduce the overlay macrocellular system performance due to increased interference caused to macrocellular users. In this article, two MIMO precoding techniques are considered at the femtocellular base stations (FBSs) to control the interference to the macrocellular users: precoding matrix index (PMI), and least interference (LI). With MIMO precoding, the limited CSI at the transmitter is the index of the precoder chosen from the codebook fed back by the receiver. The $\mathrm{LI}$ technique can be employed at the FBSs to maximize the macrocellular throughput, but it also results in significant reduction in femtocellular throughput. The PMI approach can maximize the signal power at a desired receiver, with minimal feedback. In this article, we develop algorithms that adapt at the FBSs between the LI and PMI schemes to increase both the macrocellular and femtocellular throughputs. We show that allowing for mode adaptation at each FBS improves the system performance when compared with using the same mode across the system, and a simple binary choice at each FBS can nearly achieve the optimum mode-adaptation performance. Analysis and simulation results in a multicell environment are presented to illustrate the improvement in system performance with the proposed techniques.
\end{abstract}

Keywords: MIMO precoding, Codebook, Mode adaptation, Interference, Throughput, Femtocell, Macrocell

\section{Introduction}

A femtocell is a low-power, user-deployed base station designed for indoor use. Because of their potential to provide improvement in coverage and capacity [1-3], femtocells have attracted much attention recently. The introduction of femtocells into an existing cellular system, however, also brings new challenges [4-11]. One of the most important is the interference problem, and recent articles have addressed this issue from several different perspectives. In [12], the performance of two-tier femtocellular networks with outage constraints is investigated considering cellular geometry and cross-tier interference in the downlink. In [13], the use of OFDMA is considered to cope with this interference. Optimal power allocation for femtocells is discussed in [14], based on an analysis of the macrocellular interference in OFDMA systems with fractional frequency reuse. The use of frequency scheduling to manage the co-channel and inter-carrier

*Correspondence: chenzij@udel.edu

1 Department of Electrical and Computer Engineering, University of Delaware, Newark, Delaware, USA

Full list of author information is available at the end of the article interference in OFDMA networks is studied in [15]. In [16], downlink carrier selection and transmit power calibration at the femtocells are proposed to manage interference for 3GPP systems. In [17], an uplink capacity analysis and interference avoidance strategy for a CDMA-based femtocell network is provided. Power control is used to mitigate co-channel cross-layer interference in $[18,19]$. In [18], strategies for maximum transmit power adjustment at the femtocells to suppress interference at the macrocellular base stations (MBSs) are presented, and a downlink power control strategy at the femtocells, based on a distributed utility-based, signal-to-interference-plusnoise ratio (SINR) adaptation, is proposed to alleviate the interference at the macrocell in [19]. Beam subset selection and codebook restriction are considered at the MBSs in $[20,21]$, respectively, to reduce cross-layer interference.

The motivation in this article is to improve the femtocellular system performance with MIMO precoding techniques applied at the femtocellular base stations (FBSs). MIMO precoding is one of the various closed-loop techniques adopted by IEEE 802.16e [22]. For example, we can obtain the beamforming vector for any channel matrix

\section{是 Springer}

(c) 2013 Jiang et al.: licensee Springer. This is an Open Access article distributed under the terms of the Creative Commons

Attribution License (http://creativecommons.org/licenses/by/2.0), which permits unrestricted use, distribution, and reproduction in any medium, provided the original work is properly cited. 
by finding its singular value decomposition (SVD) [23]. A scheme that quantizes the unitary beamforming matrix was presented in [24]; the collection of quantized beamforming matrices is called a codebook. The codebook is obtained by optimizing over the chordal distance or mutual information between codewords. In [25], the authors improved upon the method in [24] by maximizing the minimum chordal distance between any pair of codewords; codebooks with four antennas and feedback sizes of 3 and 6 bits are given in $[25,26]$. In this article, based on the design method in [25], codebooks for feedback sizes of 8 bits with four antennas are generated and applied in the simulation.

Also, MIMO precoding methods for interference mitigation in femtocellular systems will be studied in frequency division duplex (FDD) systems. With MIMO precoding, the receiver feeds back the index of the codeword in a codebook to the transmitter; this codeword is then applied as the precoder. With this limited CSI, it is difficult to achieve good performance for both macrocells and femtocells with the practical MIMO precoding schemes alone. Thus, we develop a mode adaptation approach at the FBSs to achieve better performance.

The transmission modes at the FBSs are adapted between least interference (LI) and precoding matrix index (PMI). The LI technique chooses the precoder at the FBS that generates the LI for the macrocellular user; this scheme maximizes the macrocellular throughput, but results in a reduction in the femtocellular throughput. The PMI approach chooses the precoder that generates the largest signal power to the femtocellular user; this maximizes the femtocellular throughput, but the interference generated to the macrocellular user is not considered.

Here, we develop a MIMO mode adaptation technique combining these two approaches to adapt the precoding mode at the FBSs and improve the system performance. A tuning factor is introduced to tune between the two modes. Two approaches, global and localized, according to the two different ways the tuning factor can be chosen, are studied and compared. With localized mode adaptation, the tuning factor is chosen independently at each FBS, while in the global approach, the same tuning factor is applied for all the FBSs. Note that the study in [27] also describes balancing the signal power at the desired receiver and the interference power at the other receivers. However, in [27], the channels are assumed to be known at the Tx and Rx, and the tuning factors are coefficients of the desired and interfering channel matrices.

\section{System overview}

A scenario with multiple macrocells and several femtocells in each macrocell is shown in Figure 1. The large hexagons represent the macrocellular coverage area, and the small circles represent the coverage areas of the individual femtocells. We assume that femtocells are deployed randomly and share the same frequency as the macrocell in each direction of transmission.

In a MIMO system with $N_{t}$ transmit and $N_{r}$ receive antennas, the received signal is

$$
\mathbf{Y}=\sqrt{P} \mathbf{H Q X}+\mathbf{n},
$$

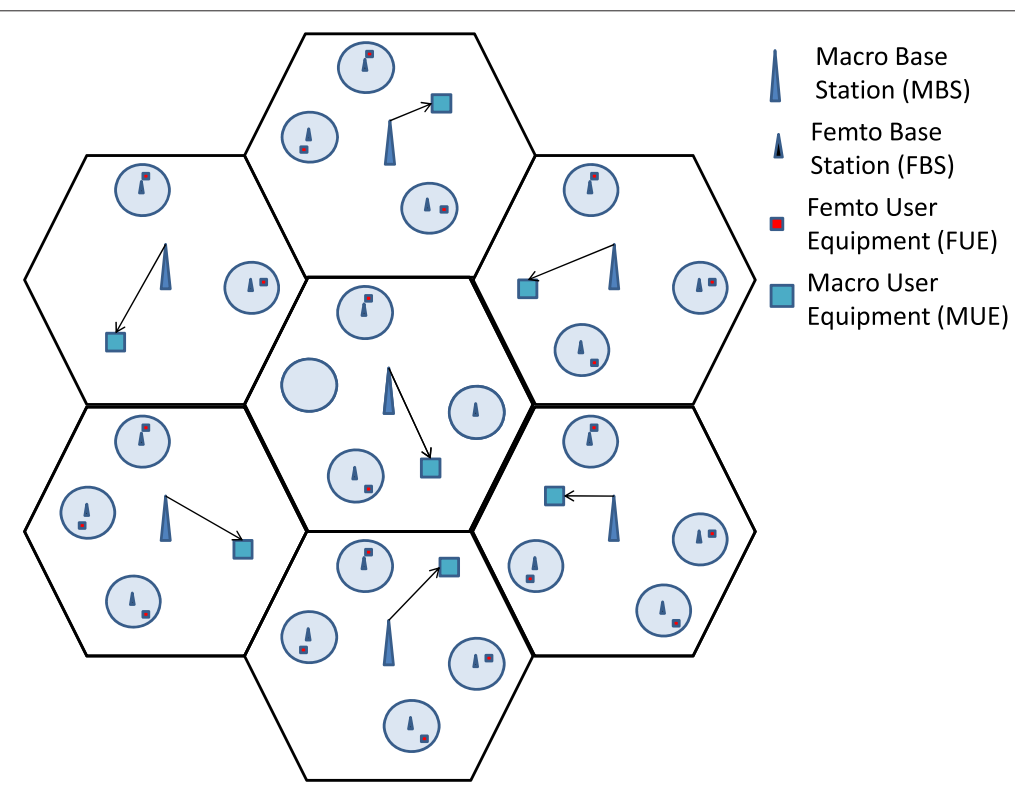

Figure 1 Macrocell/femtocellular architecture. 
where $P$ is the average received power (including path loss), $\mathbf{H}$ is the $N_{r} \times N_{t}$ channel matrix, $\mathbf{X}$ is the $N_{s} \times 1$ transmitted signal vector ( $N_{s}$ is the number of signal streams), $\mathbf{n}$ is an additive white Gaussian noise vector, and $\mathbf{Q}$ is a normalized $N_{t} \times N_{s}$ precoding matrix. In this article, we assume $N_{s}=1$, that is, a single stream is transmitted; so, $\mathbf{Q}$ is a vector. Also, it is assumed that no beamforming is done at the receivers. The channel coefficients are modeled as i.i.d. complex Gaussian random variables with zero mean and unit variance.

Consider a femtocellular system with $N_{M}$ macrocells and $N_{F}$ femtocells sharing the same frequency. Assume there is one active user equipment (UE) in each cell. Denote the channel between the $i$ th MBS/FBS and the

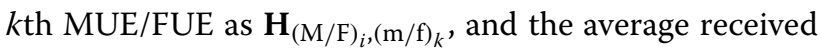
power at the $k$ th MUE/FUE from the $i$ th MBS/FBS as $P_{(\mathrm{M} / \mathrm{F})_{i},(\mathrm{~m} / \mathrm{f})_{k}}$. For example, the channel between the 2nd FBS and the 1st MUE is denoted as $\mathbf{H}_{\mathrm{F}_{2}, \mathrm{~m}_{1}}$. Then, the SINR at the MUE in the $i$ th macrocell is

$$
=\frac{\operatorname{SINR}_{m}^{(i)}}{\sum_{k=1, k \neq i}^{N_{\mathrm{M}}} P_{\mathrm{M}_{k}, \mathrm{~m}_{i}}\left\|\mathbf{H}_{\mathrm{M}_{k}, \mathrm{~m}_{i}} \mathbf{Q}_{\mathrm{M}_{k}}\right\|^{2}+\sum_{k=1}^{N_{\mathrm{F}}} P_{\mathrm{F}_{k}, \mathrm{~m}_{i}}\left\|\mathbf{H}_{\mathrm{F}_{k}, \mathrm{~m}_{i}} \mathbf{Q}_{\mathrm{F}_{k}}\right\|^{2}+\sigma_{m}^{2}},
$$

and the SINR at the FUE in the $i$ th femtocell is

$$
\begin{aligned}
& \operatorname{SINR}_{f}^{(i)} \\
& =\frac{P_{\mathrm{F}_{i}, \mathrm{f}_{i}}\left\|\mathbf{H}_{\mathrm{F}_{i}, \mathrm{f}_{i}} \mathbf{Q}_{\mathrm{F}_{i}}\right\|^{2}}{\sum_{k=1}^{N_{\mathrm{M}}} P_{\mathrm{M}_{k}, \mathrm{f}_{i}}\left\|\mathbf{H}_{\mathrm{M}_{k}, \mathrm{f}_{i}} \mathbf{Q}_{\mathrm{M}_{k}}\right\|^{2}+\sum_{k=1, k \neq i}^{N_{\mathrm{F}}} P_{\mathrm{F}_{k}, \mathrm{f}_{i}}\left\|\mathbf{H}_{\mathrm{F}_{k}, \mathrm{f}_{i}} \mathbf{Q}_{\mathrm{F}_{k}}\right\|^{2}+\sigma_{f}^{2}},
\end{aligned}
$$

where $\mathbf{Q}_{\mathrm{M}_{k}}$ and $\mathbf{Q}_{\mathrm{F}_{k}}$ are the precoders at the $k$ th $\mathrm{MBS}$ and FBS, and $\sigma_{m}^{2}$ and $\sigma_{f}^{2}$ are the noise powers at the MUE and FUE, respectively.

We consider the downlink (DL) performance of the femtocellular system, and the obtainable DL throughput is calculated using the SINR at the receiver and a fixed backoff $\delta$ from capacity. In this article, we assume that $\delta=2$ [3], reflecting what can be achieved with practical coding schemes.

\section{Codebook generation}

There are numerous ways to generate codebooks. Here, we use the method in [25] which can achieve better performance by maximizing the minimum distance between the codewords. As described in [25], the codebook is fully specified by the first codeword $\mathbf{W}_{1}$, a diagonal rotation matrix $\mathbf{G}$, and the eigen-matrix $\mathbf{M}$. The first codeword $\mathbf{W}_{1}$ is chosen to be an $N_{t} \times N_{s}$ submatrix of the $N_{t} \times N_{t}$ DFT matrix F [24]; the rotation matrix $\mathbf{G}=$ $\operatorname{diag}\left[e^{j \frac{2 \pi}{2^{L}} u_{1}}, \ldots, e^{j \frac{2 \pi}{2^{L}} u_{N_{t}}}\right]$ is specified by the number of bits per feedback $L$ and the integer vector $\mathbf{u}=\left[u_{1}, \ldots, u_{N_{t}}\right]$; and the eigen-matrix $\mathbf{M}$ is parameterized to be $\mathbf{M}=\mathbf{I}-$ $2 \mathbf{b} \mathbf{b}^{H}$, where $\mathbf{b}$ is an $N_{t} \times 1$ vector with norm 1 , and $(\cdot)^{H}$ denotes conjugate transpose. The remaining codewords are generated by $\mathbf{W}_{l}=\mathbf{M G}^{l-1} \mathbf{M}^{H} \mathbf{W}_{1}$. The codebook is optimized by maximizing the minimum chordal distance between two codewords:

$$
\mathcal{W}^{\text {opt }}\left(\mathbf{W}_{1}, \mathbf{u}, \mathbf{b}\right)=\arg \max _{\mathbf{W}_{1}, \mathbf{u}, \mathbf{b}}\left\{\min _{m, n} d_{c}\left(\mathbf{W}_{m}, \mathbf{W}_{n}\right)\right\}
$$

where $\mathbf{W}_{m}, \mathbf{W}_{n} \in \mathcal{W}\left(\mathbf{W}_{1}, \mathbf{u}, \mathbf{b}\right), \mathcal{W}\left(\mathbf{W}_{1}, \mathbf{u}, \mathbf{b}\right)$ is the codebook specified by parameters $\mathbf{W}_{1}, \mathbf{u}$, and $\mathbf{b}$, and the chordal distance is defined as [25]

$$
d_{c}\left(\mathbf{W}_{m}, \mathbf{W}_{n}\right)=1-\left\|\mathbf{W}_{m}^{H} \mathbf{W}_{n}\right\|^{2}
$$

Using the procedure described above, the codebooks for feedback sizes of 2 and 8 bits with $N_{t}=4$ and $N_{s}=1$ have

\begin{tabular}{|c|c|c|c|}
\hline L & u & b & minimum chordal distance \\
\hline \multirow[t]{4}{*}{2} & {$[1,2,4,12]$} & {$[0.3536+0.3536 \mathrm{j} ;$} & 1 \\
\hline & & $0.3536-0.3536 j ;$ & \\
\hline & & $-0.3536-0.3536 j ;$ & \\
\hline & & $-0.3536+0.3536 j]$ & \\
\hline \multirow[t]{4}{*}{3} & {$[1,2,7,6]$} & {$[0.2895+0.3635 j ;$} & 0.8282 \\
\hline & & $0.5287-0.2752 j$ & \\
\hline & & $-0.2352-0.4247 \mathrm{j} ;$ & \\
\hline & & $-0.4040+0.1729 j]$ & \\
\hline \multirow[t]{4}{*}{6} & {$[1,45,22,49]$} & {$[0.3954-0.0738 j$} & 0.3935 \\
\hline & & $0.0206+0.4326 j ;$ & \\
\hline & & $-0.1658-0.5445 j ;$ & \\
\hline & & $0.5487-0.1599 j]$ & \\
\hline \multirow[t]{4}{*}{8} & {$[1,10,102,177]$} & {$[0.4660+0.4660 j ;$} & 0.1733 \\
\hline & & $0.2827-0.2827 \mathrm{j} ;$ & \\
\hline & & $-0.1964-0.1964 j ;$ & \\
\hline & & $-0.4054+0.4054 j]$ & \\
\hline
\end{tabular}
been generated. Parameters for the codebooks are given in Table 1 . The parameters for codebooks with $\mathrm{L}=3$ and 6 bits have been taken from [25]. Next, we describe MIMO precoding methods that use these codebooks.

\section{Table 1 Codebook parameters}




\section{MIMO precoding in femtocellular systems}

Femtocells might generate significant interference to the MUEs. Although the transmit power of the femtocells is relatively low, the throughput of the MUE might degrade rapidly as the number of femtocells increases. In this section, two codebook-based methods of MIMO precoding schemes are considered at the FBSs: PMI, and LI.

\section{Precoding matrix index}

In the PMI approach, the required CSI at each FBS is the $L$-bit index of the codeword that should be applied as the precoder at the FBS and is fed back from the desired FUE. The FUE searches for the precoder from the $2^{L}$ codewords in the codebook that maximizes the signal power; for example, the precoder is determined by calculating the received SINR with each codeword applied at the FBS, that is,

$$
\mathbf{Q}_{\mathrm{PMI}}=\arg \max _{\mathbf{Q}_{c} \in \mathcal{W}}\left\|\mathbf{H}_{\mathrm{F}, \mathrm{f}} \mathbf{Q}_{c}\right\|^{2},
$$

where $\mathbf{Q}_{\text {PMI }}$ represents the chosen codeword at the FBS using the PMI technique. The $L$-bit index of this codeword could be fed back to each FBS through a local connection. This method can maximize the signal power with a given codebook and, therefore, maximize the femtocellular throughput; on the other hand, the macrocellular throughput might be reduced significantly because the interference generated to the MUE is not considered in the optimization in (6). PMI can be compared to transmit beamforming (TXBF) which maximizes the signal power without the codebook constraint.

\section{Least interference}

In the LI approach, the required CSI at each FBS is the $L$-bit index, provided by the MBS, of the codeword that should be applied as the precoder at the FBS. Here, the MUE searches for the precoder at the FBS that causes the smallest interference; for example, the precoder is determined by calculating the received SINR with each codeword applied at the FBS, that is,

$$
\mathbf{Q}_{\mathrm{LI}}=\arg \min _{\mathbf{Q}_{c} \in \mathcal{W}}\left\|\mathbf{H}_{\mathrm{F}, \mathrm{m}} \mathbf{Q}_{c}\right\|^{2},
$$

where $\mathbf{Q}_{\mathrm{LI}}$ represents the chosen codeword at the FBS using the LI technique. Similar to PMI restriction, the index of this codeword could be fed back to the MBS and then shared with the FBS through a local connection. This method can minimize the interference at the MUE from the FBSs and, therefore, maximize the macrocellular throughput; on the other hand, the femtocellular throughput might be reduced significantly because the signal power is not considered in the optimization in (7). LI can be compared to zero-forcing (ZF) which minimizes the interference power without the codebook constraint.

\section{Mode adaptation with MIMO precoding}

As stated above, the PMI and LI techniques each have their specific advantages, but each alone is not sufficient to obtain good system performance. PMI maximizes the femtocellular throughput but results in poor macrocellular performance; LI maximizes the macrocellular throughput but degrades the femtocellular performance severely. Therefore, here, we consider mode adaptation (MA) that combines PMI and LI to obtain the "best" system performance.

\section{System performance}

The motivation for combining PMI and LI at the FBSs is to improve the system performance. Note that different system requirements give different criteria for performance. When considering both the macrocellular and femtocellular performance, one possible system performance metric is the sum-rate of all the users in the cell, including macrocellular and femtocellular users. However, in most cases, there will be many more FBSs and FUEs than MUEs at any instant in time; thus, maximizing the sum-rate of all the users will lead to good femtocellular performance but poor macrocellular throughput. Another potential system performance metric is the weighted sum of the average macrocellular and femtocellular throughputs. But, maximizing the sum of the average throughputs does not guarantee fairness among users.

If we consider a proportional fairness constraint [28], the objective function could be the product of the average throughputs of all the users. Since we assume all the femtocells work in the same way and have the same average throughput, and the macrocellular and femtocellular throughputs might be of different importance, here, we define the system performance metric as

$$
T_{g}=T_{m}^{\eta} \cdot T_{f}^{1-\eta}
$$

where $T_{m}$ and $T_{f}$ represent the average macrocellular and femtocellular throughputs, respectively, and $0 \leq \eta \leq 1$ is the weight given to $T_{m} . T_{g}$ represents the weighted geometric mean of the macrocellular and femtocellular throughputs. When $\eta=0, T_{g}=T_{f}$, which means the femtocellular throughput will be maximized, and PMI will be applied at all the FBSs; when $\eta=1, T_{g}=T_{m}$, meaning the macrocellular throughput will be maximized, and LI will be applied at all the FBSs. 


\section{Mode adaptation algorithm}

With MIMO precoding, the only CSI available at the FBS are the indices of the precoding vectors $\mathbf{Q}_{\mathrm{PMI}}$ and $\mathbf{Q}_{\mathrm{LI}}$; thus, the FBS must adapt its MIMO mode based on this information. The chordal distance between a codeword in the codebook $\mathbf{Q}_{c}$ and the PMI vector $\mathbf{Q}_{P M I}$ is

$$
d_{\mathrm{PMI}}\left(\mathbf{Q}_{c}\right)=1-\left\|\mathbf{Q}_{\mathrm{PMI}}^{H} \mathbf{Q}_{c}\right\|^{2},
$$

and between $\mathbf{Q}_{c}$ and the $\mathrm{LI}$ vector $\mathbf{Q}_{\mathrm{LI}}$ is

$$
d_{\mathrm{LI}}\left(\mathbf{Q}_{c}\right)=1-\left\|\mathbf{Q}_{\mathrm{LI}}^{H} \mathbf{Q}_{c}\right\|^{2} .
$$

We show in Appendix 1 that, when the channels are not known and only $\mathbf{Q}_{\mathrm{PMI}}$ and $\mathbf{Q}_{\mathrm{LI}}$ are available, the SINRs at the desired and undesired users can be improved by minimizing the chordal distances $d_{\mathrm{PMI}}$ and $d_{\mathrm{LI}}$, respectively. One possible hybrid is to minimize a linear combination of $d_{\mathrm{PMI}}$ and $d_{\mathrm{LI}}$. We can define the hybrid mode adaptation vector $\mathbf{Q}_{\mathrm{MA}}$ as

$$
\mathbf{Q}_{\mathrm{MA}}=\arg \min _{\mathbf{Q}_{c} \in \mathcal{W}}\left\{c \cdot d_{\mathrm{PMI}}\left(\mathbf{Q}_{c}\right)+(1-c) \cdot d_{\mathrm{LI}}\left(\mathbf{Q}_{c}\right)\right\}
$$

where $0 \leq c \leq 1$ is a tuning factor reflecting the relative importance of increasing the signal power at the desired user versus reducing the interference power at the undesired user.

The optimization in (11), however, requires calculation of the chordal distances and a search over the entire codebook, which might be difficult at the FBSs, especially with a large codebook. In order to reduce the complexity, we instead consider a linear combination of the precoding vectors

$$
\mathbf{Q}_{\mathrm{MA}}=\frac{\lambda \mathbf{Q}_{\mathrm{PMI}}+(1-\lambda) \mathbf{Q}_{\mathrm{LI}}}{\left\|\lambda \mathbf{Q}_{\mathrm{PMI}}+(1-\lambda) \mathbf{Q}_{\mathrm{LI}}\right\|} .
$$

Since the required information is the same, this simplified method should have the same mode adaptation ability. Given a value of $c$ in (11), we can get a corresponding tuning factor $\lambda$ in (12), $\lambda=\arg \max _{0 \leq \lambda \leq 1} U(\lambda)(0 \leq \lambda \leq$ 1), where

$$
U(\lambda)=c\left\|\mathbf{Q}_{\mathrm{PMI}}^{H} \mathbf{Q}_{\mathrm{MA}}\right\|^{2}+(1-c)\left\|\mathbf{Q}_{\mathrm{LI}}^{H} \mathbf{Q}_{\mathrm{MA}}\right\|^{2} .
$$

Setting $U^{\prime}(\lambda)=0$, and after some manipulations, we get the following

(i) When $c=\frac{1}{2}, \lambda=\frac{1}{2}$;

(ii) When $c \neq \frac{1}{2}$,

$$
\lambda=\frac{\sqrt{c(1-c) \operatorname{Tr}\{\Theta\}^{2}+(2 c-1)^{2}}-c \operatorname{Tr}\{\Theta\}+2 c-1}{(2 c-1)(2-\operatorname{Tr}\{\Theta\})},
$$

where $\Theta=\mathbf{Q}_{\mathrm{PMI}} \mathbf{Q}_{\mathrm{LI}}^{H}+\mathbf{Q}_{\mathrm{LI}} \mathbf{Q}_{\mathrm{PMI}}^{H}$, and $\operatorname{Tr}\{\cdot\}$ represents the trace of a square matrix. If $\lambda<0$, set $\lambda=0$; if $\lambda>1$, set $\lambda=1$.

With the same tuning function, the simplified MA algorithm reduces the complexity compared with the original one. When $\lambda=0, \mathbf{Q}_{\mathrm{MA}}=\mathbf{Q}_{\mathrm{LI}}$; when $\lambda=1, \mathbf{Q}_{\mathrm{MA}}=$ $\mathbf{Q}_{\text {PMI }}$; and when $\lambda$ is between 0 and 1 , the precoding mode at the FBS is tuned between LI and PMI. How to select the optimum $\lambda$ will be addressed later based on the system performance metric.

The MA algorithm is described as follows:

1. The FUE determines the $\mathbf{Q}_{\mathrm{PMI}}$ at the FBS which generates the largest signal power at the FUE, and feeds back the L-bit index of the $\mathbf{Q}_{\mathrm{PMI}}$ to the FBS.

2. The MUE determines the $\mathbf{Q}_{\mathrm{LI}}$ at the FBS which generates the LI at the MUE, and feeds back the L-bit index of the $\mathbf{Q}_{\mathrm{LI}}$ to the MBS. The MBS shares this information with the FBS.

3. The FBS chooses the precoder according to (12) (with an appropriate choice of $\lambda$ ).

Note that after each FBS chooses the precoder, the FUE and MUE estimate the SINR and feed back the information to the base stations. Using this information, the base stations employ adaptive modulation and coding (AMC) to achieve a throughput close to the channel capacity.

\section{Global MA}

In the global MA approach, we assume that $\lambda$ is determined on a system-wide level, and all the FBSs in the same macrocell apply the same tuning factor. Assume that the average SINRs at the MUE and the FUE can be estimated centrally and are known to the FBSs, then the average macrocellular $\left(T_{m}\right)$ and femtocellular throughputs $\left(T_{f}\right)$ can be estimated. The tuning factor $\lambda$ can then be optimized over an objective function containing both $T_{m}$ and $T_{f}$, which reflects the system requirement. Here, we use (8) as the system performance metric.

\section{Localized MA}

Global MA requires system-wide information, which might be difficult to obtain in practice. In addition, using the same $\lambda$ at all the FBSs lacks flexibility. Therefore, we also consider a localized MA approach; in this case, each FBS independently chooses its tuning factor $\lambda$ according to its specific situation.

The simplest form of mode adaptation at the FBSs is to apply binary MA. In this case, the value of the tuning factor $\lambda$ is either 0 or 1 , i.e., the precoding mode at each FBS is either LI or PMI. By observing (14), we can also find that the probability of $\lambda=0$ or $\lambda=1$ is quite high, which 
indicates that binary MA can also achieve good system performance. In the following, we consider two methods of localized binary MA, one is based on path loss, and the other one is based on distance.

\section{Decision based on long-term performance observation}

Which precoding mode is used by the FBS depends on the interference power it generates to the MUE. The average interference power received at the MUE from the FBS is determined by the transmit power and the long-term fading characteristics. We assume the transmit power of the FBSs is unchanged. Since the shadow and multipath fading are stochastic, the average path loss between the FBS and the MUE determines the average received interference power at the MUE from the FBS. Thus, here, we consider mode adaptation at the FBSs based on the average path loss between the FBS and the MUE.

We assume the uplink transmit power of the MUE is known to the FBS, and the FBS can measure the received uplink power from the MUE. Then, the average uplink (UL) path loss between the MUE and the FBS, PLUL, can be obtained. This average path loss reflects the distance between the FBS and the MUE. The average DL path loss, $\mathrm{PL}_{\mathrm{DL}}$, is proportional to $\mathrm{PL}_{\mathrm{UL}}$, so the UL path loss reflects the average interference power at the MUE from the FBS. Here, we consider this UL path-loss information to determine the value of $\lambda$ and the precoding mode at each FBS. The FBS can apply PMI $(\lambda=1)$ if the average path loss is larger than a specified threshold; otherwise $\operatorname{LI}(\lambda=0)$ is used. The optimal path-loss threshold can be estimated through long-term observation of the performance.

Note that we can also use a continuous value of $\lambda$, independently at each FBS, but, as we will show in the next section, using only a binary value for $\lambda$ at the FBSs can achieve almost the same performance as continuous $\lambda$.

\section{Decision based on distance}

With the localized binary MA decision based on longterm performance observation, the optimal path-loss threshold needs to be estimated through long-term measurement and observation, which might be difficult to implement in practice. Therefore, here we consider another binary MA approach based on the distance between the FBS and the MUE, and assume that the location of the MUE can be obtained for example using GPS, which is widely available in many terminals, like smartphones. This method avoids the search for the optimum path-loss threshold, which may be difficult to estimate. Each FBS only needs to obtain the distribution of the distance between the MUE and itself. Since the location of each FBS is fixed, this distribution can be acquired at each FBS by long-term measurement.
Generally, longer distance results in larger path loss. We assume that the transmit power at each FBS is fixed, then the average interference power received at the MUE from the FBS can be determined by the distance between the FBS and the MUE. Given the distance threshold $d_{\mathrm{th}}$, when the distance between the FBS and the MUE $d<d_{\text {th }}$, LI should be applied; otherwise, PMI is applied at the FBS. In order to achieve the optimum system performance, we need to determine the distance threshold $d_{\mathrm{th}}$ for each FBS.

The FBSs and the MUE are usually randomly distributed in the macrocell. Let $p$ denote the probability of the LI scheme being applied at a FBS. Therefore, $\operatorname{Pr}\left\{d<d_{\text {th }}\right\}=$ $\operatorname{Pr}\{\mathrm{LI}\}=p$, and $\operatorname{Pr}\left\{d \geq d_{\mathrm{th}}\right\}=\operatorname{Pr}\{\mathrm{PMI}\}=1-p$. So optimizing $d_{\text {th }}$ is equivalent to optimizing $p$. The derivation of the value of $p$ is given in Appendix 2 .

The location of each FBS is fixed, and the location of the active MUE is random. Assume that the distribution of the distance between the FBS and the MUE is available at each FBS, the optimum distance threshold $d_{\text {th }}$ at each FBS depends on the distribution of the distance between the FBS and the MUE. Since $\operatorname{Pr}\left\{d<d_{\text {th }}\right\}=\operatorname{Pr}\{\mathrm{LI}\}=p$, the value of the optimum $d_{\text {th }}$ for the $k$ th FBS should be chosen so that $d_{t h, k}=F_{k}^{-1}(p)$, where $F_{k}$ is the CDF of the distance between the MUE and the $k$ th FBS.

The localized binary MA algorithm based on distance can be summarized as follows:

1. By long-term observation, the MBS measures the average macrocellular and femtocellular throughputs when all the FBSs apply PMI; and then measures the average macrocellular and femtocellular throughputs when all the FBSs apply LI.

2. Each FBS obtains the distribution of the distance between the FBS and the MUE by long-term observation.

3. The MBS calculates the value of $p$ according to (31) for a given $\eta$, and then shares this information and the location of the MUE with all the FBSs.

4. Each FBS calculates the distance threshold $d_{t h, k}$, using $p$ and the distribution of the distance, then calculates the distance between the FBS and the MUE at each time instant, and chooses the appropriate value of $\lambda$.

\section{Simulation results}

Assume that there are three sectors in each macrocell, one active MUE in each sector, and one active FUE in each femtocell at each time instant. Also, assume that the MBSs transmit without using beamforming. Path-loss models follow those in $[29,30]$, and the key parameters are given in Table 2. The femtocells are uniformly distributed in the macrocell, and each UE is also uniformly distributed inside the coverage area of its serving BS. 
Table 2 Simulation parameters

\begin{tabular}{cc}
\hline $\begin{array}{c}\text { Number of transmit antennas } N_{t} \\
\text { Carrier frequency } f_{C}\end{array}$ & 4 \\
Macro BS antenna height & $2.5 \mathrm{GHz}$ \\
above mean rooftop level $\Delta h_{B S}$ & \\
\# of MUE per sector & $20 \mathrm{~m}$ \\
\# of FUE per femtocell & 1 \\
Macro BS transmit power & 1 \\
Macrocell radius & $46 \mathrm{dBm}$ \\
Femtocell radius & $1000 \mathrm{~m}$ \\
Power spectral density of noise & $10 \mathrm{~m}$ \\
Bandwidth & $-174 \mathrm{dBm} / \mathrm{Hz}$ \\
\hline
\end{tabular}

\section{Performance of MIMO precoding algorithms}

In this subsection, the performance of the MIMO precoding schemes PMI and LI will be illustrated with the macrocellular and femtocellular throughputs (in bits/ $\mathrm{sec} / \mathrm{Hz}$ ) at the 50th percentile of the CDF (50\% throughputs) as a function of the number of femtocells per macrocell sector.

Figures 2 and 3 show the macrocellular and femtocellular 50\% DL throughputs, respectively, with PMI and LI applied at the FBSs. Different sizes of codebooks are used for comparison. PMI and LI are compared with the corresponding unquantized transmission schemes, TXBF and $\mathrm{ZF}$, respectively. Figure 2 shows that the macrocellular performance degrades as the number of FBSs increases due to the increased interference power received at the MUE from the FBSs. PMI and TXBF at the FBSs both achieve the same macrocellular performance as not using $\mathrm{BF}$. This is because the interference power generated to

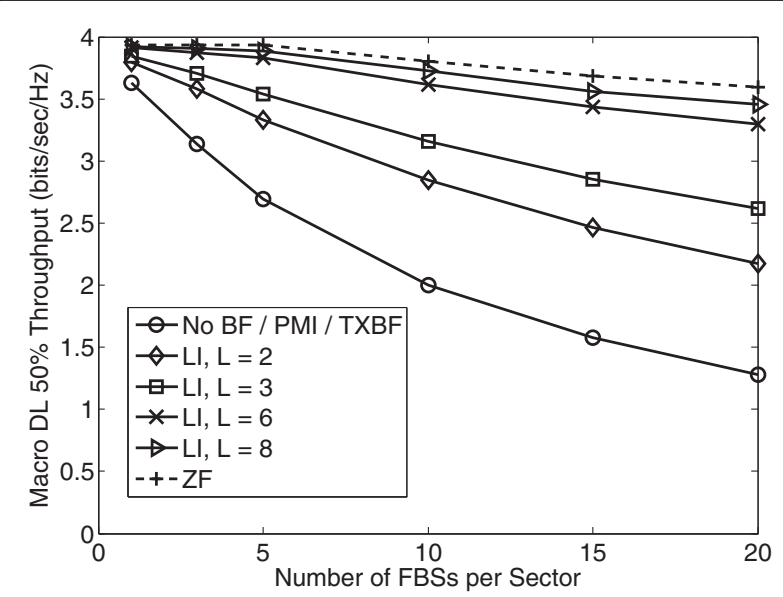

Figure 2 50\% macrocellular DL throughputs with PMI and LI applied at the FBSs. FBS transmit power is $10 \mathrm{dBm}$.

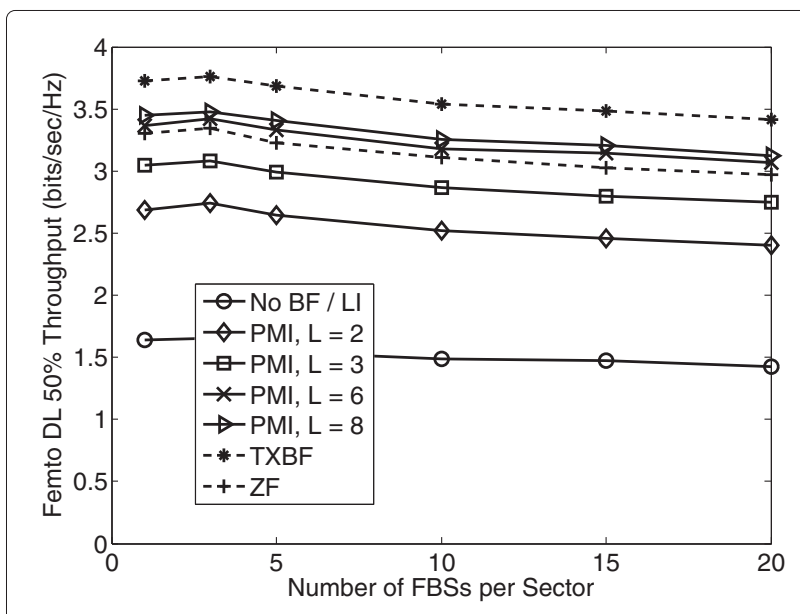

Figure $3 \mathbf{5 0 \%}$ femtocellular DL throughputs with PMI and LI applied at the FBSs. FBS transmit power is $10 \mathrm{dBm}$.

the MUE is not considered with PMI and TXBF, and the received interference power at the MUE from the FBSs is the same on average. The macrocellular throughput with LI is better than when no BF is used at the FBSs because the interference power is reduced. Since ZF minimizes the interference power at the MUE from the FBSs, ZF works the best for macrocellular performance. The LI scheme reduces the interference power at the MUE to a minimum with the given limited options, thus, there is some performance degradation compared with ZF. However, as the size of the codebook and the number of precoder options increases, the macrocellular performance with LI approaches that of the unquantized ZF scheme.

Figure 3 shows that the femtocellular performance does not degrade much as the number of FBSs increases because of the low transmit power of the FBSs. LI at the FBSs achieves the same femtocellular performance as not doing beamforming. This is because the signal power at the FUE is not considered in the LI approach, and the average signal power with LI is the same as that without BF. Better femtocellular performance can be obtained with PMI applied at the FBSs. The femtocellular throughput with PMI approaches that for unquantized TXBF as the size of the codebook increases. Performance with ZF applied at the FBSs is also fairly good, because here we assume each FBS is equipped with four transmit antennas and it is only necessary to eliminate the interference generated to the MUE in the same macrocell; thus the rest of the degrees of freedom can be utilized to increase the signal power at the FUE.

Similar trends are observed for the throughputs at the 10th percentile of the CDF (indicative of cell-edge performance). The figure is not shown here due to space limitation. 


\section{Performance of mode adaptation algorithms}

Since a larger codebook is required for better tuning, an 8 -bit codebook is used. Here, we also assume that there are $10 \mathrm{FBSs}$ in each macrocell sector.

\section{Performance of global and localized ma}

In Figure 4, we demonstrate, using the 50th-percentile throughput, the effect of the tuning factor $\lambda$ on the macrocellular and femtocellular throughputs using global MA. The transmit power at the FBSs is $10 \mathrm{dBm}$. As $\lambda$ increases, the precoding mode at the FBSs is tuned from LI to PMI. Consequently, the macrocellular throughput is reduced, and the femtocellular throughput is increased, accordingly.

Figure 5 shows the optimized 50\% macrocellular and femtocellular throughputs with global MA applied at the FBSs as the FBS transmit power is varied. The system performance $T_{g}(8)$ is maximized with an exhaustive search over $\lambda$. We know that PMI at the FBSs achieves the best femtocellular performance but the worst macrocellular performance, and LI works in the opposite way; therefore, the performances of PMI and LI serve as bounds for the femtocellular and macrocellular throughputs. With global MA, when the FBS transmit power is low, PMI is mainly applied at the FBSs; and when the FBS transmit power is high, LI is employed to avoid severe interference at the MUE from the FBSs. Since the same value of $\lambda$ is applied at all the FBSs, in most cases, the mode at all the FBSs is either PMI or LI. Thus, the precoding mode at the FBSs is not efficiently adapted.

Figure 6 shows the optimized 50\% macrocellular and femtocellular throughputs with localized binary MA

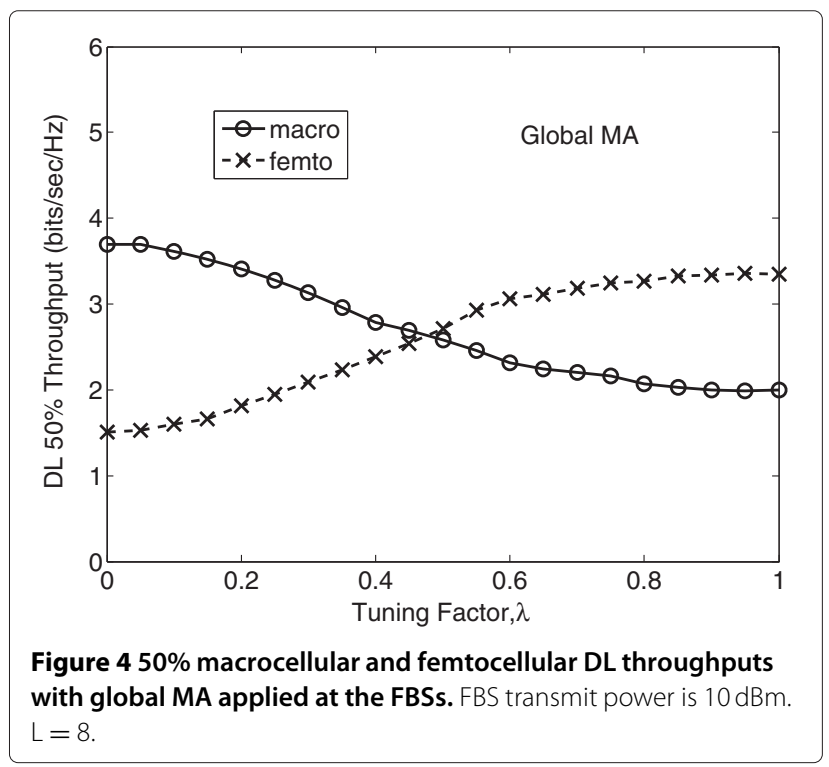

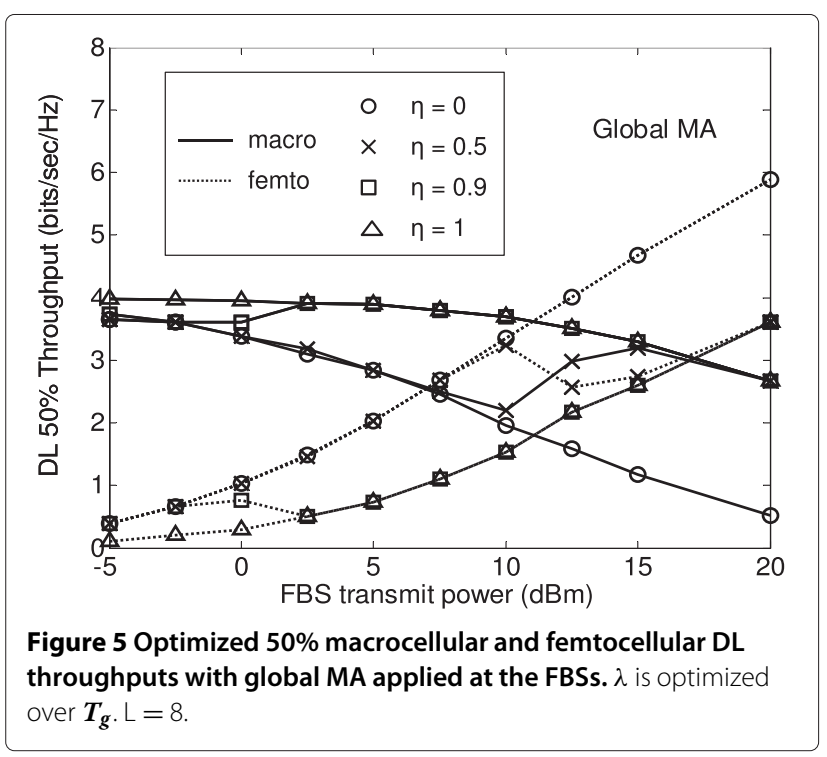

based on long-term performance observation (LPO) applied at the FBSs; $T_{g}(8)$ is also optimized with an exhaustive search over a reasonable range of the pathloss threshold, which is determined by the coverage area of the MBSs. The curves in Figure 6 are smoother than those with global MA because the precoding mode at each FBS is more efficiently and properly adapted. As $\eta$ increases, more importance is given to the macrocell, so the optimized macrocellular throughput improves and the femtocellular throughput is reduced. Overall, the system performance is improved with localized MA.

Figure 7 shows the optimized 50\% macrocellular and femtocellular throughputs with localized binary MA

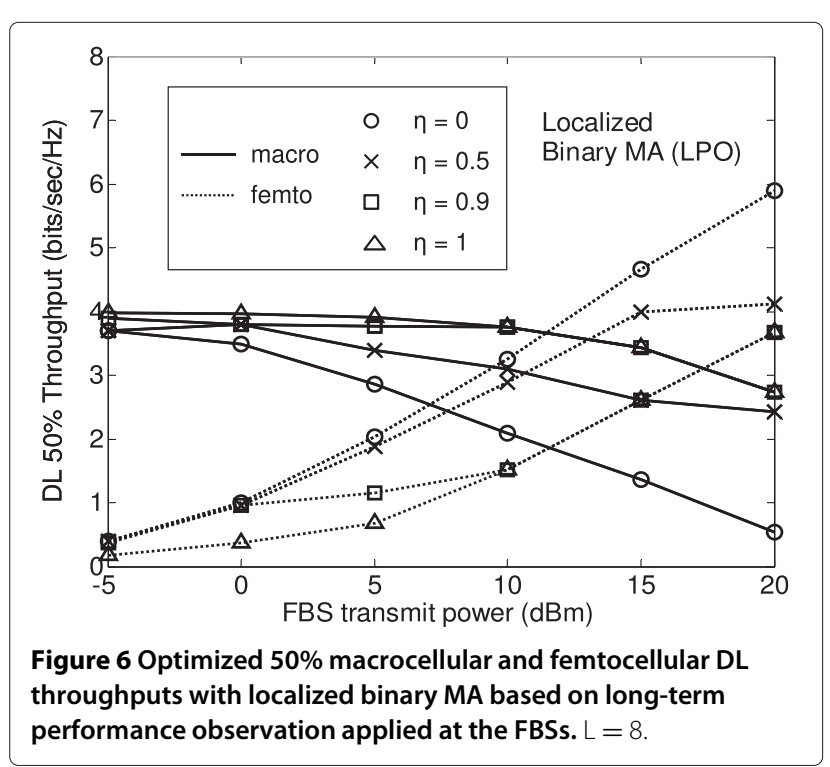




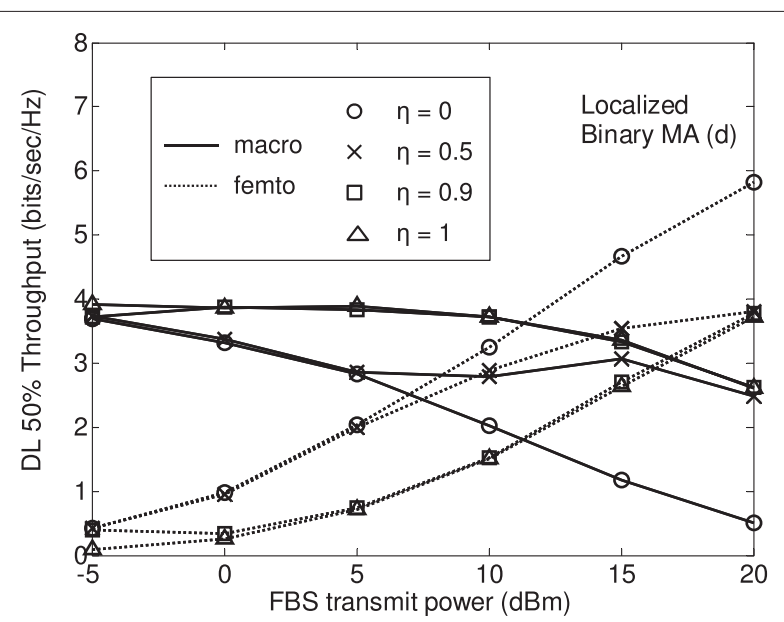

Figure 7 Optimized $50 \%$ macrocellular and femtocellular DL throughputs with localized binary MA based on distance applied at the FBSs. $L=8$.

based on distance, applied at the FBSs. The precoding mode is independently chosen at each FBS according to the distribution of the distance between the FBS and the MUE. It shows that binary MA based on distance can also efficiently adapt the mode at each FBS. Due to the approximation in the analysis, however, binary MA based on distance does not work as well as binary MA based on uplink path loss, which used an exhaustive search to find the optimum path-loss threshold and is not practical.

\section{Performance comparison}

Figure 8 shows the maximum $T_{g}$ obtainable with different precoding schemes at the FBSs for $\eta=0.5$, that is, the

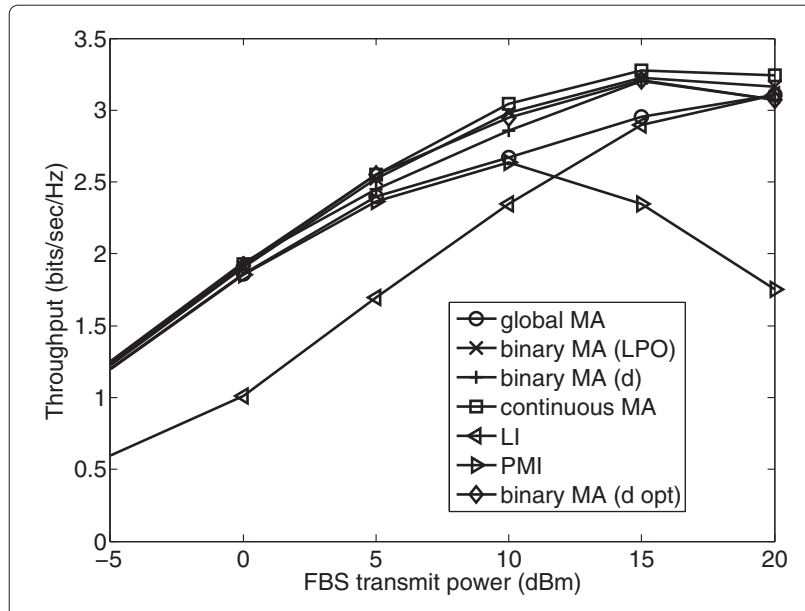

Figure 8 Maximum $T_{g}$ achievable $(\boldsymbol{\eta}=\mathbf{0 . 5}) . \mathrm{L}=8$. macrocellular and femtocellular performances are equally weighted. With the localized continuous MA approach, the $\lambda$ at each FBS is independently optimized to maximize the weighted combination of the received SINRs at the MUE and the FUE. This method requires perfect CSI, and therefore can serve as an upper bound on the modeadaptation performance. Note that binary MA suffers only a small penalty compared with continuous MA, but it is much simpler to implement. With binary MA based on distance with optimal $p$, the value of $p$ is obtained by a brute-force search over the range from 0 to 1 , which is not practical. We can also observe that the performance with suboptimal $p$ is close to that with optimal $p$, but with much reduced complexity.

The MA technique combines PMI and LI, and adapts the mode at the FBSs between the two; thus, MA has better system performance than either PMI or LI. The system performance is further improved using localized MA. With low FBS transmit power, the interference at the MUE from the FBSs is small, and the precoding modes at the FBSs are mainly PMI. As the FBS transmit power increases, $T_{g}$ initially improves because of the increased femtocellular throughput, and then degrades due to the reduced macrocellular throughput. At the high end of the FBS transmit power, LI outperforms PMI because LI avoids severe degradation of the macrocellular performance, and the femtocellular throughput is compensated by the high transmit power. PMI at the FBSs, in this case, leads to seriously degraded macrocellular throughput. The performance of the MA techniques will converge to that of LI as the FBS transmit power is further increased. This is because, with high FBS transmit power, the precoding mode at the FBSs reverts to LI in order to get better system performance.

Figures 9 and 10 show the optimized 50\% macrocellular and femtocellular DL throughputs, respectively, with different precoding modes at the FBSs. We observe that when the FBS transmit power is lower, PMI is applied at the FBSs with global MA; this reduces the macrocellular throughput. On the other hand, with localized MA, the precoding mode is chosen according to the specific situation at each FBS. Thus, localized MA improves the macrocellular performance without degrading the femtocellular performance much when the FBS transmit power is lower. When the FBS transmit power increases, LI is applied at the FBSs with global MA, and localized MA leads to improvement in femtocellular performance with more flexibility. Similar trends are observed for $10 \%$ throughput as shown in Figure 11.

\section{Conclusions}

In this study, codebook-based precoding methods of interference mitigation in femtocellular systems are 


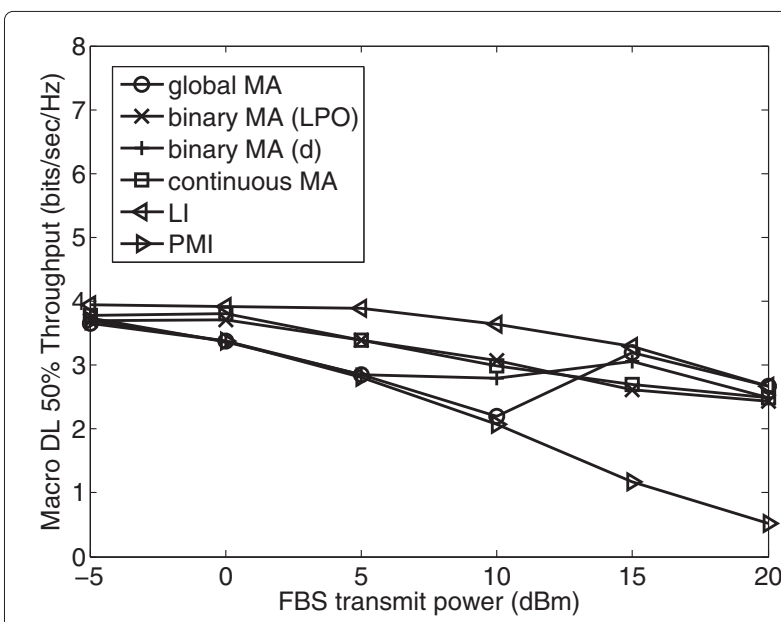

Figure 9 Optimized 50\% macrocellular DL throughput with different precoding modes applied at the FBSs $(\boldsymbol{\eta}=\mathbf{0 . 5}) . \mathrm{L}=8$.

considered. Different sizes of codebooks are generated and applied to these precoding methods. In general, LI maximizes the macrocellular throughput, but leads to low femtocellular throughput; and PMI achieves the maximum femtocellular throughput but poor macrocellular performance. Based on this, we considered mode adaptation at the FBSs in order to improve both the macrocellular and femtocellular throughputs and obtain better femtocellular system performance. The precoding mode is adapted at the FBSs between PMI and LI. Global and localized approaches were considered and compared. We showed that mode adaptation at the FBSs brings performance gain to the femtocellular system, and the system performance can be further improved by localized mode adaptation with more flexibility at each FBS. We also

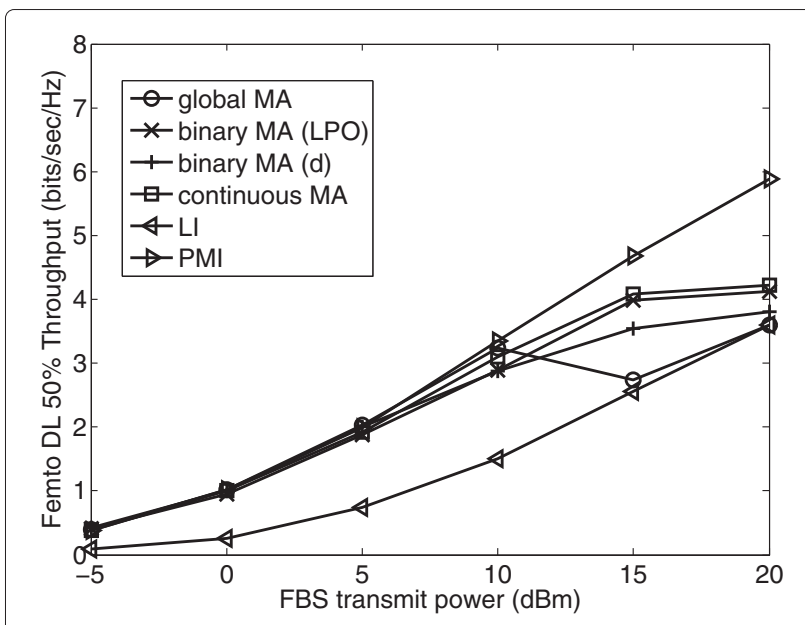

Figure 10 Optimized $50 \%$ femtocellular DL throughput with different precoding modes applied at the FBSs $(\boldsymbol{\eta}=\mathbf{0 . 5}) . L=8$.

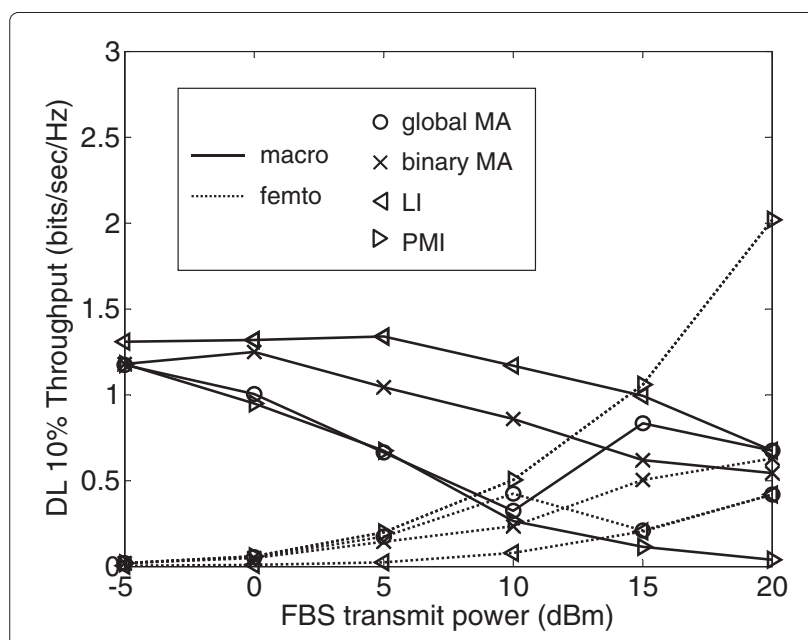

Figure 11 Optimized $10 \%$ macrocellular and femtocellular DL throughputs with different precoding modes applied at the FBSs $(\boldsymbol{\eta}=\mathbf{0 . 5}) . \mathrm{L}=8$.

showed that a simple localized binary choice at each FBS can provide good performance for both the macrocellular and femtocellular users, and nearly achieve the upper bound on the performance of the mode adaptation approach.

In summary, we have the following points on precoding based mode adaptation in femtocells:

- When the FBS is close to the MUE or the channel gain from the FBS and the MUE is high, LI should be applied at the FBS to minimize the interference power from the FBS to the MUE.

- When the FBS is far from the MUE or the channel between the FBS and the MUE is in deep fading, the interference is small, so PMI should be applied at the FBS to maximize the signal power from the FBS to the FUE.

- For all other cases, the tuning factor should be calculated using the mode adaptation algorithms to tune between PMI and LI.

More efficient and practical methods for choosing the optimum $\lambda$ will be studied in the future. In addition, a comparison of the MA algorithm with other interference management schemes, e.g., enhanced inter-cell interference coordination (eICIC) in 3GPP, will also be investigated.

\section{Appendix 1}

\section{Chordal distance $d_{\mathrm{PMI}}$ and $d_{\mathrm{LI}}$}

In the MIMO system described in Section II, the received power from the transmitter is $\|\mathbf{H Q}\|^{2}$. Using SVD, $\mathbf{H}$ can be represented as $\mathbf{H}=\mathbf{U} \Sigma \mathbf{V}^{H}$, where $\mathbf{U}$ and $\mathbf{V}$ are unitary 
matrices; and $\|\mathbf{H Q}\|^{2}=\sum_{i=1}^{N_{r}} \sigma_{i}^{2}\left\|\mathbf{Q}^{H} \mathbf{v}_{i}\right\|^{2}$, where $\sigma_{i}$ is the $i$ th singular value of $\mathbf{H}$, and $\mathbf{v}_{i}$ is the $i$ th column of $\mathbf{V}$. Considering (6), and denoting $\mathbf{H}_{D}$ as the desired channel, we have

$$
\left\|\mathbf{H}_{D} \mathbf{Q}_{c}\right\|^{2}=\sum_{i=1}^{N_{r}} \sigma_{i}^{2}\left\|\mathbf{Q}_{c}^{H} \mathbf{v}_{i}\right\|^{2} \leq \sum_{i=1}^{N_{r}} \sigma_{i}^{2}\left\|\mathbf{Q}_{P M I}^{H} \mathbf{v}_{i}\right\|^{2} .
$$

Since $\left\|\mathbf{Q}_{\mathrm{PMI}}^{H} \mathbf{v}_{i}\right\|^{2} \leq 1,\left\|\mathbf{Q}_{c}^{H} \mathbf{v}_{i}\right\|$ and $\left\|\mathbf{Q}_{c}^{H} \mathbf{Q}_{\mathrm{PMI}}\right\|$ have the same distribution, given $\mathbf{v}_{i}$,

$$
\lim _{\alpha \rightarrow+\infty} \operatorname{Pr}\left\{\frac{\left\|\mathbf{Q}_{c}^{H} \mathbf{v}_{i}\right\|^{2}}{\left\|\mathbf{Q}_{\mathrm{PMI}}^{H} \mathbf{v}_{i}\right\|^{2}}-\left\|\mathbf{Q}_{c}^{H} \mathbf{Q}_{\mathrm{PMI}}\right\|^{\alpha} \geq 0\right\}=1 .
$$

Thus, $\left\|\mathbf{Q}_{c}^{H} \mathbf{v}_{i}\right\|^{2} \geq\left\|\mathbf{Q}_{\mathrm{PMI}}^{H} \mathbf{v}_{i}\right\|^{2}\left\|\mathbf{Q}_{c}^{H} \mathbf{Q}_{\mathrm{PMI}}\right\|^{\alpha}$, as long as $\alpha$ is large enough. Combining (15) and (16), and denoting $S_{\max }=\sum_{i=1}^{N_{r}} \sigma_{i}^{2}\left\|\mathbf{Q}_{\mathrm{PMI}}^{H} \mathbf{v}_{i}\right\|^{2}$,

$$
\left\|\mathbf{Q}_{c}^{H} \mathbf{Q}_{\mathrm{PMI}}\right\|^{\alpha} S_{\max } \leq\left\|\mathbf{H}_{D} \mathbf{Q}_{c}\right\|^{2} \leq S_{\max }
$$

equality is achieved iff. $\mathbf{Q}_{c}=\mathbf{Q}_{\mathrm{PMI}}$. Given a channel matrix $\mathbf{H}_{D}$ and a codebook, $S_{\max }$ is determined. However, in the case that $\mathbf{H}_{D}$ is not known and only $\mathbf{Q}_{\mathrm{PMI}}$ is available, if $\mathbf{Q}_{c} \neq \mathbf{Q}_{P M I}$, we can improve the received signal power $\left\|\mathbf{H}_{D} \mathbf{Q}_{c}\right\|^{2}$ by choosing the $\mathbf{Q}_{c}$ that maximizes $\left\|\mathbf{Q}_{c}^{H} \mathbf{Q}_{\mathrm{PMI}}\right\|$, or minimizes $d_{\mathrm{PMI}}\left(\mathbf{Q}_{c}\right)=1-\left\|\mathbf{Q}_{c}^{H} \mathbf{Q}_{\mathrm{PMI}}\right\|^{2}$.

Considering (7), we have

$$
\left\|\mathbf{H}_{I} \mathbf{Q}_{c}\right\|^{2}=\sum_{i=1}^{N_{r}} \sigma_{i}^{2}\left\|\mathbf{Q}_{c}^{H} \mathbf{v}_{i}\right\|^{2} \geq \sum_{i=1}^{N_{r}} \sigma_{i}^{2}\left\|\mathbf{Q}_{\mathrm{LI}}^{H} \mathbf{v}_{i}\right\|^{2},
$$

where $\mathbf{H}_{I}$ is the interference channel. Also, we have

$$
\lim _{\beta \rightarrow+\infty} \operatorname{Pr}\left\{\left\|\mathbf{Q}_{c}^{H} \mathbf{v}_{i}\right\|^{2}\left\|\mathbf{Q}_{L I}^{H} \mathbf{v}_{i}\right\|^{2}+\left\|\mathbf{Q}_{c}^{H} \mathbf{Q}_{\mathrm{LI}}\right\|^{\beta} \leq 1\right\}=1 .
$$

Thus, $\left\|\mathbf{Q}_{c}^{H} \mathbf{v}_{i}\right\|^{2} \leq \frac{1-\left\|\mathbf{Q}_{c}^{H} \mathbf{Q}_{\mathrm{LI}}\right\|^{\beta}}{\left\|\mathbf{Q}_{L I}^{H} \mathbf{v}_{i}\right\|^{2}}$, as long as $\beta$ is large enough. Combining (18) and (19), and denoting $I_{\min }=$ $\left\|\mathbf{H}_{I} \mathbf{Q}_{\mathrm{LI}}\right\|^{2} \sum_{i=1}^{N_{r}} \sigma_{i}^{2}\left\|\mathbf{Q}_{\mathrm{LI}}^{H} \mathbf{v}_{i}\right\|^{2}$, we obtain

$$
I_{\min } \leq\left\|\mathbf{H}_{I} \mathbf{Q}_{c}\right\|^{2} \leq\left(1-\left\|\mathbf{Q}_{c}^{H} \mathbf{Q}_{\mathrm{LI}}\right\|^{b}\right) \sum_{i=1}^{N_{r}} \frac{\sigma_{i}^{2}}{\left\|\mathbf{Q}_{\mathrm{LI}}^{H} \mathbf{v}_{i}\right\|^{2}}
$$

equality is achieved iff. $I_{\min }=0$ and $\mathbf{Q}_{c}=\mathbf{Q}_{\mathrm{LI}}$. Given a channel matrix $\mathbf{H}_{I}$ and a codebook, $\sum_{i=1}^{N_{r}} \frac{\sigma_{i}^{2}}{\left\|\mathbf{Q}_{\mathrm{LI}}^{H} \mathbf{v}_{i}\right\|^{2}}$ is determined. However, in the case that $\mathbf{H}_{I}$ is not known and only $\mathbf{Q}_{\mathrm{LI}}$ is available, if $\mathbf{Q}_{c} \neq \mathbf{Q}_{L I}$, we can reduce the received interference power $\left\|\mathbf{H}_{I} \mathbf{Q}_{c}\right\|^{2}$ by choosing the $\mathbf{Q}_{c}$ that maximizes $\left\|\mathbf{Q}_{c}^{H} \mathbf{Q}_{\mathrm{LI}}\right\|$, or minimizes $d_{\mathrm{LI}}\left(\mathbf{Q}_{c}\right)=$ $1-\left\|\mathbf{Q}_{c}^{H} \mathbf{Q}_{\mathrm{LI}}\right\|^{2}$

\section{Appendix 2}

\section{Derivation of the value of $p$}

The femtocellular throughput mainly depends on the MIMO mode of the FBS and the signal power at the FUE. The MIMO mode adopted at the FBSs does not change the interference power received at the FUE. So, the average femtocellular throughput with binary mode adaptation at each FBS can be written as

$$
T_{f}=p \cdot T_{f}^{\mathrm{LI}}+(1-p) \cdot T_{f}^{\mathrm{PMI}}
$$

where $T_{f}^{\mathrm{LI}}$ is the average femtocellular throughput when $\mathrm{LI}$ is applied at the FBS, and $T_{f}^{\mathrm{LI}}$ is the average femtocellular throughput when PMI is applied at the FBS.

The MIMO mode at each FBS determines the interference power received at the MUE; therefore, we cannot obtain the average macrocellular throughput in a simple and direct way, as for (21). The average macrocellular throughput is an integral over all possible locations of the MUE. Denote the channels between the MUE and all the transmitters (including the serving and interfering MBSs and all the FBSs) as $\{\mathbf{H}\}$, then the signal and interference powers received at the MUE all depend on $\{\mathbf{H}\}$. Denote the interference power received at the MUE from the $k$ th FBS transmitting in LI mode as $P_{F, m}^{\mathrm{LI}(k)}$, and the interference power received at the MUE from the $k$ th FBS transmitting in PMI mode as $P_{F, m}^{\mathrm{PMI}(k)}$. So, the total interference power at the MUE is

$$
\begin{aligned}
P_{I, m}(\{\mathbf{H}\})= & \sum_{k=1}^{N_{F}}\left[p \cdot P_{F, m}^{\mathrm{LI}(k)}(\{\mathbf{H}\})+(1-p) \cdot P_{F, m}^{\mathrm{PMI}(k)}(\{\mathbf{H}\})\right] \\
& +P_{0}(\{\mathbf{H}\}),
\end{aligned}
$$

where $N_{F}$ is the number of femtocells in the macrocell, and $P_{0}(\{\mathbf{H}\})$ is the interference power at the MUE from other macrocells. So, the average macrocellular throughput can be written as

$$
T_{m}=\int_{\{\mathbf{H}\}} f(\{\mathbf{H}\}) T_{\mathrm{MUE}}(\{\mathbf{H}\}) d(\{\mathbf{H}\})
$$


where

$$
T_{\mathrm{MUE}}(\{\mathbf{H}\})=\log _{2}\left\{1+\frac{P_{S, m}(\{\mathbf{H}\})}{\sum_{k=1}^{N_{F}}\left[p \cdot P_{F, m}^{\mathrm{LI}(k)}(\{\mathbf{H}\})+(1-p) \cdot P_{F, m}^{\mathrm{PMI}(k)}(\{\mathbf{H}\})\right]+P_{0}(\{\mathbf{H}\})+\sigma_{m}^{2}}\right\}
$$

is the instantaneous macrocellular throughput dependent on the channels, $f(\{\mathbf{H}\})$ is the distribution of all the channels related to the MUE, $P_{S, m}(\{\mathbf{H}\})$ and $\sigma_{m}^{2}$ are respectively, the signal and noise power received at the MUE.

It is difficult to obtain $f(\{\mathbf{H}\})$, therefore, it is difficult to obtain a closed-form expression for the average macrocellular throughput $T_{m}$ in (23). To simplify the problem, in the following, we will instead find the solution that maximizes a close upper bound of the average system performance $T_{g}$. The upper bound can be obtained from the property of convex functions.

Since $f(x)=\log _{2}\left(1+\frac{a}{x+b}\right)$ is a convex function of $x$, we have

$$
\begin{aligned}
& \log _{2}\left\{1+\frac{P_{S, m}(\{\mathbf{H}\})}{\sum_{k=1}^{N_{F}}\left[p \cdot P_{F, m}^{\mathrm{LI}(k)}(\{\mathbf{H}\})+(1-p) \cdot P_{F, m}^{\mathrm{PMI}(k)}(\{\mathbf{H}\})\right]+P_{0}(\{\mathbf{H}\})+\sigma_{m}^{2}}\right\} \leq p . \\
& \log _{2}\left[1+\frac{P_{S, m}(\{\mathbf{H}\})}{\sum_{k=1}^{N_{F}} P_{F, m}^{\mathrm{LI}(k)}(\{\mathbf{H}\})+P_{0}(\{\mathbf{H}\})+\sigma_{m}^{2}}\right]+(1-p) \cdot \log _{2}\left[1+\frac{P_{S, m}(\{\mathbf{H}\})}{\sum_{k=1}^{N_{F}} P_{F, m}^{\mathrm{PMI}(k)}(\{\mathbf{H}\})+P_{0}(\{\mathbf{H}\})+\sigma_{m}^{2}}\right],
\end{aligned}
$$

and

$$
\begin{aligned}
T_{m} \leq & p \cdot \int_{\{\mathbf{H}\}} f(\{\mathbf{H}\}) \log _{2}\left[1+\frac{P_{S, m}(\{\mathbf{H}\})}{\sum_{k=1}^{N_{F}} P_{F, m}^{\mathrm{LI}(k)}(\{\mathbf{H}\})+P_{0}(\{\mathbf{H}\})+\sigma_{m}^{2}}\right] d(\{\mathbf{H}\}) \\
& +(1-p) \cdot \int_{\{\mathbf{H}\}} f(\{\mathbf{H}\}) \log _{2}\left[1+\frac{P_{S, m}(\{\mathbf{H}\})}{\sum_{k=1}^{N_{F}} P_{F, m}^{\mathrm{PMI}(k)}(\{\mathbf{H}\})+P_{0}(\{\mathbf{H}\})+\sigma_{m}^{2}}\right] d(\{\mathbf{H}\}) \\
= & p \cdot T_{m}^{\mathrm{LI}}+(1-p) \cdot T_{m}^{\mathrm{PMI}},
\end{aligned}
$$

where $T_{m}^{\mathrm{LI}}$ represents the average macrocellular throughput when all FBSs apply LI, and $T_{m}^{\mathrm{PMI}}$ represents the average macrocellular throughput when all FBSs apply PMI. Denote $T_{m}^{\mathrm{UB}}=p \cdot T_{m}^{\mathrm{LI}}+(1-p) \cdot T_{m}^{\mathrm{PMI}}$. Since both $T_{m}^{\mathrm{UB}}$ and $T_{m}$ are increasing functions of $p$, increasing $T_{m}^{\mathrm{UB}}$ by changing the value of $p$ will also increase $T_{m}$.

Therefore, the system performance metric is

$$
\begin{aligned}
T_{g} & =T_{m}^{\eta} \cdot T_{f}^{1-\eta} \\
& \leq\left[p \cdot T_{m}^{\mathrm{LI}}+(1-p) \cdot T_{m}^{\mathrm{PMI}}\right]^{\eta} \cdot\left[p \cdot T_{f}^{\mathrm{LI}}+(1-p) \cdot T_{f}^{\mathrm{PMI}}\right]^{1-\eta} .
\end{aligned}
$$

Denote

$$
T_{g}^{\mathrm{UB}}=\left[p \cdot T_{m}^{\mathrm{LI}}+(1-p) \cdot T_{m}^{\mathrm{PMI}}\right]^{\eta} \cdot\left[p \cdot T_{f}^{\mathrm{LI}}+(1-p) \cdot T_{f}^{\mathrm{PMI}}\right]^{1-\eta} .
$$

Since this upper bound is obtained from the property of convex functions, it is not a loose bound. By optimizing this upper bound, we can obtain a suboptimal value of $p$ which can achieve similar performance as the optimal $p$. This can also be shown with simulation results.

The derivative of $T_{g}^{\mathrm{UB}}$ can be easily computed as

$$
T_{g}^{\mathrm{UB}^{\prime}}(p)=\eta \cdot\left[\frac{p \cdot T_{f}^{\mathrm{LI}}+(1-p) \cdot T_{f}^{\mathrm{PMI}}}{p \cdot T_{m}^{\mathrm{LI}}+(1-p) \cdot T_{m}^{\mathrm{PMI}}}\right]^{1-\eta} \cdot\left(T_{m}^{\mathrm{LI}}-T_{m}^{\mathrm{PMI}}\right)-(1-\eta) \cdot\left[\frac{p \cdot T_{m}^{\mathrm{LI}}+(1-p) \cdot T_{m}^{\mathrm{PMI}}}{p \cdot T_{f}^{\mathrm{LI}}+(1-p) \cdot T_{f}^{\mathrm{PMI}}}\right]^{\eta} \cdot\left(T_{f}^{\mathrm{PMI}}-T_{f}^{\mathrm{LI}}\right) .
$$


By setting $T_{g}^{\mathrm{UB}^{\prime}}(p)=0$, we obtain the optimum value of $p$

$$
p=\frac{\eta \cdot T_{m}^{\mathrm{LI}} T_{f}^{\mathrm{PMI}}+(1-\eta) \cdot T_{m}^{\mathrm{PMI}} T_{f}^{\mathrm{LI}}-T_{m}^{\mathrm{PMI}} T_{f}^{\mathrm{PMI}}}{\left(T_{m}^{\mathrm{LI}}-T_{m}^{\mathrm{PMI}}\right)\left(T_{f}^{\mathrm{PMI}}-T_{f}^{\mathrm{LI}}\right)}
$$

Denote $\Delta T_{m}=T_{m}^{\mathrm{LI}}-T_{m}^{\mathrm{PMI}}$ and $\Delta T_{f}=T_{f}^{\mathrm{PMI}}-$ $T_{f}^{\mathrm{LI}}$, representing the difference between the maximum and minimum average macrocellular and femtocellular throughputs, respectively. Denote $T_{p}^{\mathrm{max}}=T_{m}^{\mathrm{LI}} T_{f}^{\mathrm{PMI}}$ and $T_{p}^{\min }=T_{m}^{\mathrm{PMI}} T_{f}^{\mathrm{LI}}$, representing the maximum and minimum products of the average macrocellular and femtocellular throughputs, respectively, and denote $T_{p}^{\mathrm{PMI}}=$ $T_{m}^{\mathrm{PMI}} T_{f}^{\mathrm{PMI}}$ representing the product of the average macrocellular and femtocellular throughputs when PMI is applied at all the FBSs. Then, the optimum value of the probability of LI applied can be written as

$$
p=\operatorname{Pr}\{\mathrm{LI}\}=\frac{\eta T_{p}^{\max }+(1-\eta) T_{p}^{\min }-T_{p}^{\mathrm{PMI}}}{\Delta T_{m} \Delta T_{f}} .
$$

Since $0 \leq p \leq 1$, if $p<0$, set $p=0$; if $p>1$, set $p=1$.

\section{Competing interests}

The authors declare that they have no competing interests.

\section{Acknowledgements}

This study was supported by a grant provided by the Intel Corporation, and US National Science Foundation (NSF) under Grant No. 1017053.

\section{Author details}

${ }^{1}$ Department of Electrical and Computer Engineering, University of Delaware, Newark, Delaware, USA. ${ }^{2}$ Wireless Commun. Lab., Intel Labs, Intel Corporation, Santa Clara, California, USA

Received: 2 August 2012 Accepted: 17 December 2012 Published: 23 January 2013

\section{References}

1. V Chandrasekhar, I Andrews, A Gatherer, Femtocell network: a survey. IEEE Commun. Mag. 46(9), 59-67 (2008)

2. S Yeh, S Talwar, S Lee, H Kim, WiMAX femtocells: a perspective on network architecture, capacity, and coverage. IEEE Commun. Mag. 46(10), 58-65 (2008)

3. H Claussen, AL Swindon, in Proc. of IEEE PIMRC. Performance of macroand co-channel femtocells in a hierarchical cell structure (Athens, Greece, 2007), pp. 1-5

4. I Guvenc, Statistics of macrocell-synchronous femtocell-asynchronous users' delays for improved femtocell uplink receiver design. IEEE Commun. Lett. 13(4), 239-241 (2009)

5. RY Kim, JS Kwak, KEtemad, WiMAX femtocell: requirements, challenges, and solutions. IEEE Commun. Mag. 47(9), 84-91 (2009)

6. A Golaup, M Mustapha, LB Patanapongpibul, Femtocell access control strategy in UMTS and LTE. IEEE Commun. Mag. 47(9), 117-123 (2009)

7. HS Jo, C Mun, J Moon, J Yook, Self-optimized coverage coordination in femtocell networks. IEEE Trans. Wirel. Commun. 9(10), 2977-2982 (2010)

8. J Xiang, Y Zhang, T Skeie, L Xie, Downlink spectrum sharing for cognitive radio femtocell networks. IEEE Systs. J. 4(4), 524-534 (2010)

9. G Gur, S Bayhan, F Alagoz, Cognitive femtocell networks: an overlay architecture for localized dynamic spetrum access. IEEE Wirel. Commun. 17(4), 62-70 (2010)
10. K Lee, O Jo, DH Cho, Cooperative resource allocation for guaranteeing intercell fairness in femtocell networks. IEEE Commun. Lett. 15(2), 214-216 (2011)

11. CH Ko, HY Wei, On-demand resource-sharing mechanism design in two-tier OFDMA femtocell networks. IEEE Trans. Veh. Tech. 60(2), 1059-1071 (2011)

12. Y Kim, S Lee, D Hong, Performance analysis of two-tier femtocell networks with outage constraints. IEEE Trans. Wirel. Commun. 9(9), 2695-2700 (2010)

13. DL Perez, A Valcarce, G Roche, J Zhang, OFDMA femtocells: a roadmap on interference avoidance. IEEE Commun. Mag. 47(9), 41-48 (2009)

14. JY Lee, SJ Bae, YM Kwon, MY Chung, Interference analysis for femtocell deployment in OFDMA systems based on fractional frequency reuse. IEEE Commun. Lett. 15(4), 425-427 (2011)

15. ME Sahin, I Guvenc, M Jeong, H Arslan, Handling, CCl and ICI in OFDMA femtocell networks through frequency scheduling. IEEE Trans. Consumer Electron. 55(4), 1936-1944 (2009)

16. M Yavuz, F Meshkati, S Nanda, A Pokhariyal, N Johnson, B Raghothaman, A Richardson, Interference management and performance analysis of UMTS/HSPA + femtocells. IEEE Commun. Mag. 47(9), 102-109 (2009)

17. V Chandrasekhar, JG Andrews, Uplink capacity and interference avoidance for two-tier femtocell networks. IEEE Trans. Wirel. Commun. 8(7), 3498-3509 (2009)

18. H Jo, C Mun, J Moon, J Yook, Interference mitigation using uplink power control for two-tier femtocell networks. IEEE Trans. Wirel. Commun. 8(10), 4906-4910 (2009)

19. V Chandrasekhar, JG Andrews, T Muharemovic, Z Sheng, A Gatherer, Power control in two-tier femtocell networks. IEEE Trans. Wirel. Commun. 8(8), 4316-4328 (2009)

20. S Park, W Seo, Y Kim, S Lim, D Hong, Beam subset selection strategy for interference reduction in two-tier femtocell networks. IEEE Trans. Wirel. Commun. 9(11), 3440-3449 (2010)

21. S Park, W Seo, S Choi, D Hong, A beamforming codebook restriction for cross-tier interference coordination in two-tier femtocell networks. IEEE Trans. Veh. Tech. 60(4), 1651-1663 (2011)

22. IEEE Std 802.16e-2005 and IEEE Std 802.16-2004/Cor 1-2005

23. A Goldsmith, Wireless Communications. (Cambridge University Press, New York, 2005)

24. BM Hochwald, TL Marzetta, TJ Richardson, W Sweldens, R Urbanke Systematic design of unitary space-time constellations. IEEE Trans. Info. Theory. 46(6), 1962-1973 (2000)

25. Q Li, XE Lin, JC Zhang, MIMO precoding in 802.16e WiMAX. J. Commun. Netws. 9(2), 141-149 (2007)

26. IEEE P802.16Rev2/D9. http://www.ieee802.org/16/pubs/80216Rev2.htm

27. ZKM Ho, D Gesbert, in Proc. of IEEE International Conf. Commun. (ICC). Balancing egoism and altruism on interference channel: the MIMO case (Cape Town, South Africa, 2010), pp. 1-5

28. R Mazumdar, LG Mason, C Douligieris, Fairness in network optimal flow control: optimality of product forms. IEEE Trans. Commun. 39(5), 775-782 (1991)

29. Report ITU-r m.2135, Guidelines for evaluation of radio interface technologies for IMT-advanced. http://www.itu.int/dms_pub/itu-r/opb/ rep/R-REP-M.2135-2008-PDF-E.pdf

30. IST-4-027756 WINNER II deliverable d1.1.2 v1.2. http://www.cept.org/files/ 1050/documents/winner2

doi:10.1186/1687-1499-2013-12

Cite this article as: Jiang et al:: MIMO precoding and mode adaptation in femtocellular systems. EURASIP Journal on Wireless Communications and Networking 2013 2013:12. 